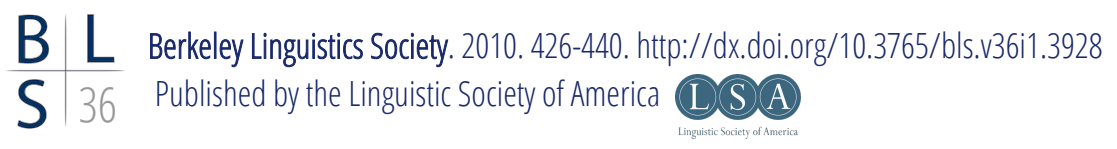

\title{
Neighborhood Density in Phonological Alternations
}

\author{
SVERRE STAUSLAND JOHNSEN \\ Harvard University
}

\section{Introduction}

Neighborhood density (ND) is a measure of how similar a word is to other words in the lexicon. In response to the growing evidence of the significance of ND for word identification and word production, linguists have started asking what role ND could play in phonological alternations, without reaching a consensus.

This paper assesses the role of ND in a phonological alternation in Norwegian, by asking whether the alternation is best predicted by ND or by the phonological structure of the alternators. The results reveal how these factors are strongly correlated and equally good predictors of alternations.

A question previously not addressed in the literature is what role ND could play for novel words in phonological alternations. An experiment conducted with nonce forms shows that ND cannot predict speakers' production, whereas the phonological structure of the alternators can. Based on this discrepancy between existing and novel words, I conclude that although the observed effect of ND in word identification can affect how words are phonologically represented over time, it plays no role in the mapping from representation to surface realization. ${ }^{1}$

\section{$1 \quad$ Norwegian retroflexion}

Norwegian distinguishes an alveolar laminal /s/ from a postalveolar apical 'retroflex'/s/ in virtually all positions (see Kristoffersen 2000:22f. and Simonsen \&

\footnotetext{
${ }^{1}$ This paper has benefited from comments and suggestions by Adam Albright, Michael Becker, and the audiences at the MIT Phonology Circle and the 2009 LSA Meeting. I am grateful to the people who volunteered to participate in the experiments, and to Vegard Johnsen for helping me recruit them. I am especially grateful to Kjetil Aasen for volunteering to tag the tokens analyzed in this paper. The raw data from the experiments reported in this paper can be found on the author's website.
} 
Moen 2004:610ff. for the articulation): ${ }^{2}$
(1) /se:/ 'see'
/š:/ 'happen'
word-initial prevocalic
(2) /suæ:t/ 'very'
/suæ:t/ 'huge'
word-initial preconsonantal
(3) $/$ tosk/ 'fool'
/tosk/ 'cod'
(4) /farsi/ 'Farsi'
/kirș/ 'kirsch'
postvocalic preconsonantal
(5) /ma:s/ 'nagging'
/ma:s/ 'Mars'
postconsonantal
word-final postvocalic
(6) /lays/ 'along'
/re'vays/ 'revenge'
word-final postconsonantal

The contrast is rare in some contexts (such as (2), (4), and (6)) where the contrast exists thanks to incorporated loanwords from other languages or idiosyncratic native developments, but it is nevertheless invariably present for such words.

When a word beginning in $/ \mathrm{s} /$ is directly preceded by a morpheme ending in the apical alveolar tap /r/ (see Kristoffersen 2000:24 and Moen et al. 2003:1756 for its articulation), the tap regularly deletes (Kristoffersen 2000:315), and /s/ surfaces as a retroflex [s]. The retroflexion of $/ \mathrm{s} /$ to [s] occurs both with words in prevocalic/s/ (7-8) and preconsonantal/s/ (9-11):
(7) /somər-su:r/ >
[soməsu:t] 'summer sun'
(8) /somər-sy:n/ >
[soməsy:n] 'summer vision'
(9) /somər-spil/ > [soməspil] 'summer games'
(10) /somər-stœ:v/ > [soməstœ:v] 'summer dust'
(11) /somər-sku:/ >
[soməsku:] 'summer shoes'

Despite earlier descriptions of the retroflex rule as being obligatory (Eliasson 1986:282, Kristoffersen 2000:316f., Torp 2007:70), the final step of the process described above $(/ \mathrm{s} />[\mathrm{s}])$ is in fact variably applied. According to intuition (which is verified in the following sections), retroflexion is more commonly applied to words in preconsonantal /s/ than to prevocalic /s/. For the examples above, retroflexion is strongly dispreferred in (7), where [soməsu:c] is the favored realization, but strongly preferred for (11). No words, however, are obligatorily exempted from or obligatorily included in the retroflex rule.

\section{$2 \quad$ Predicting Alternations}

A challenge for phonologists when dealing with optional processes with much variation is to pin down the predictive factors for when the process does or does not apply, and to understand why those factors affect the process in the way they do. A recent proposal by Ussishkin \& Wedel (2009) highlights the possibility that

\footnotetext{
${ }^{2}$ Throughout this paper, 'Norwegian' will be used as a convenient short term for 'Urban East Norwegian' (see Kristoffersen 2000:8).
} 


\section{Neighborhood Density in Phonological Alternations}

this could in part be determined by the relationship between words in the lexicon. They draw attention to the fact that for many phonological alternations, there is a negative correlation between the likelihood of a word to alternate and how many similar words there are in the language - the word's neighborhood density (ND). Since participants in experiments do worse in recognizing words that have undergone phonological alternations (Tsapkini et al. 1999, Järvikivi et al. 2009:227ff.) or that reside in dense neighborhoods (Luce \& Pisoni 1998:20, 24f., Dirks et al. 2001:6ff.), Ussishkin \& Wedel point out that words that both have a dense neighborhood and also participate in an alternation are at a double disadvantage for lexical access. As a way to make lexical access more effective, they suggest that phonological alternations are suppressed in dense neighborhoods (2009:280ff.).

Centering the discussion on the variation in final devoicing in Turkish, Pycha et al. (2007) and Becker \& Nevins (2009) evaluate Ussishkin \& Wedel's claim by doing statistical analyses on the Turkish lexicon, concluding that no such significant correlation can be found, and that ND in general plays no role in phonological alternations. The Turkish alternation is in their view best described by referral to the word size, measured in morae or syllables.

The role of ND can similarly be assessed for the variable application of retroflexion in Norwegian. By gathering data on applied retroflexion for words with prevocalic /s/ (simple onset) and preconsonantal /s/ (complex onset), a statistical analysis can be performed to evaluate whether the retroflexion of a word is best predicted by referring to its ND or to its onset structure.

\section{Experiment 1}

To evaluate the role of ND and onset structure (Onset) for Norwegian retroflexion, a production experiment was designed with words beginning with simple and complex onsets. Since the two commonest word initial/s/-onsets in Norwegian are simple /s-/ and /st-/, /st-/ was chosen as the complex onset. Five nouns in simple /s-/ and five nouns in /st-/ were selected for production, all taken from the 15 most frequent monosyllabic nouns in /s-/ in the LBK corpus. ${ }^{3}$ All ten items had a / $\mathrm{V}(\mathrm{V}) \mathrm{C} /$ rhyme.

The ND for these ten words were found by automatically generating a list of all possible neighbors of their retroflex variants, since these are the forms that suffer from the 'double disadvantage' identified by Ussishkin \& Wedel (2009), further filtered through hand-crafted phonotactic surface constraints. Following the methodological principle outlined in Pycha et al. (2007:370, 377), a neighbor was defined as any phonological word in this list present in the author's native

\footnotetext{
${ }^{3}$ Leksikografisk bokmålskorpus, with ca. 40 million tagged tokens. http://www.hf.uio.no/tekstlab/ English/corpora.html
} 
lexicon.

In the experiment, these ten nouns formed nominal compounds of the kind in (7-11) with a nonce first element bemmer-, ending in the retroflex trigger $/ \mathrm{f} /$, and which was assigned no semantic content. The items were appropriately illustrated to ensure that the participants treated them as the intended high-frequency nouns.

Ten native speakers of Norwegian with a median and mean age of 30 participated in the experiment. All items were presented in randomized order for each participant, with multiple instances of each item embedded in a frame story, interspersed among filler items. A pilot study revealed that speakers often hyperarticulate tokens for some time at the beginning of an experiment, at which register retroflexion is almost always absent. Since such speech is of no interest in this study, each participant's production up until the first instance of applied retroflexion was treated as a warm-up to the experiment, and the items contained in the warm-up were then repeated at the end of the experiment.

The participants' production was recorded, and each item was tagged as retroflex or non-retroflex independently by the author and by a phonetically trained linguist who is a native speaker of Norwegian, and who was kept unaware of the purpose and design of the experiment. Only items that were tagged identically by both were included in the final analysis.

$0.29 \%$ of the items were excluded due to erroneous and disfluent production, and a subsequent $0.41 \%$ were excluded due to disagreement between the two taggers. In the end, the data set contained 2406 trials. Overall, $60.89 \%$ of the tokens underwent retroflexion, and every item in the experiment exhibited variation. The item with least retroflexion was /sur// 'sun', with $29 \%$, and the item with most retroflexion was /stof/ 'material' with $86 \%$.

\subsection{Results}

Figure (12) shows the relation between ND and retroflexion, and figure (13) shows the relation between Onset and retroflexion. Both figures confirm the expected tendencies of correlations in that there is less retroflexion for words in dense neighborhoods and for words in a simple /s-/ onset. 
(12)

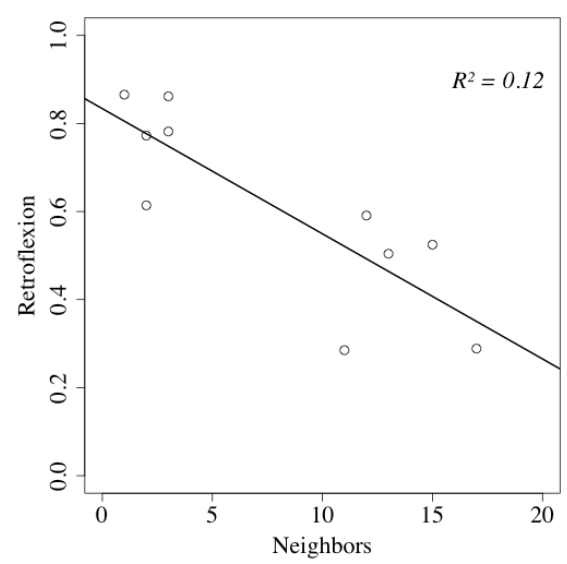

(13)

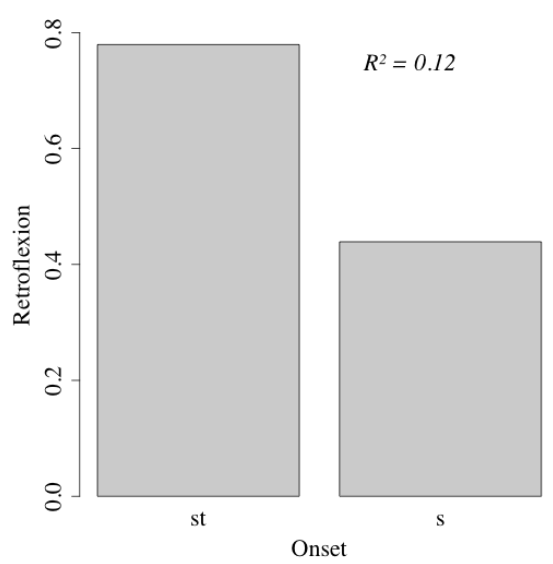

The results were analyzed with generalized linear mixed effects models (Jaeger 2008:442ff.) using maximum likelihood fitting in R (Bates \& Maechler 2010, R development core team 2010). ${ }^{4}$ The binomially distributed dependent variable is the presence or absence of retroflexion. Among the independent variables, subjects and words were treated as random effects (Baayen et al. 2008). Fixed effects were ND, Onset, the log lemma frequency of the word in the LBK corpus, and various factors related to the experimental setting that are of no interest in this study. The significance of the fixed effects was estimated with likelihood ratio tests. $^{5}$

When ND was not included in the analysis, Onset was a highly significant factor $\left(\chi^{2}(1)=12.35, p<.001\right)$, and similarly when Onset was not included in the analysis, ND was highly significant $\left(\chi^{2}(1)=11.71, p<.001\right)$. Log frequency proved insignificant $\left(\chi^{2}(1)=.54, p=.46\right)$. Onset and ND are, however, tightly correlated with each other, $r(2404)=-.96, p<.001$, since words in a simple $/ \mathrm{s}-/$ consistently have more neighbors than words with a complex /sC-/ onset. In figure (12) above, the circles with a ND $>10$ represent the five words in a simple /s-/, whereas the circles with a ND $<5$ represent the five words in /st-/. When the residuals of ND are added to a model with Onset included, no significant improvement is made $\left(\chi^{2}(1)=.21, p=.65\right)$. The same holds when the residuals of Onset are added to a model with ND included $\left(\chi^{2}(1)=.85, p=.36\right)$. This means that ND and Onset explain the same data equally well, and neither is able to

\footnotetext{
${ }^{4}$ The models were fit with maximum likelihood fitting in order to allow likelihood ratio tests of the fixed effects (cf. Pinheiro \& Bates 2000:76).

${ }^{5}$ Applying likelihood ratio tests to fixed effects in these mixed models has its limitations (Pinheiro \& Bates 2000:87ff.), and should ideally be replaced by probability estimates using Markov chain Monte Carlo sampling (Baayen et al. 2008:396ff.). Since it is currently unclear how such sampling should be performed in generalized linear mixed models, likelihood ratio tests are nevertheless our best approximation of probability estimates of fixed effects (Bates 2008).
} 
explain any data the other one does not also explain. ${ }^{6}$

\subsection{Discussion}

The results from experiment 1 are inconclusive with respect to the effect of ND in the retroflex alternation in Norwegian, since the analysis is not able to tease apart the role played by ND and the role played by Onset. When it comes to the question of why ND or Onset would have the observed effect on the retroflexion rate in the first place, Ussishkin \& Wedel (2009:280ff.) make the suggestion that the grammar suppresses alternations in dense neighborhoods in order to achieve more efficient lexical access. Building on the same observations as Ussishkin \& Wedel do, however, it is possible to obtain the same correlation between ND and phonological alternations without assuming that the grammar optimizes in a teleological fashion under functional pressure.

First, it is observed that adult speakers change their phonology to better reflect the ambient language. Longitudinal studies have shown how their phonology changes in line with phonological changes in the speech community (Harrington et al. 2000, Sankoff \& Blondeau 2007:570ff.). Experimental studies agree, revealing how speakers immediately change their production to better match recently experienced stimuli (Goldinger 1998:257ff., Nielsen 2008:31f.), an effect that persists well beyond the experimental setting (Goldinger 2000:157).

Second, phonology has been shown to be word-specific. For example, the realization of a certain phoneme might depend on the word it belongs to. A particularly well studied case is vowel reduction in English, where it has been found that words in dense neighborhoods are pronounced with less reduction in their vowels than words in sparse neighborhoods (Wright 2003:81f., Stephenson 2004:367, Munson \& Solomon 2004:1055). Behavioral experiments support this finding, as it has been shown that participants unconsciously mimic the phonetic properties of recently perceived words significantly more than words they did not recently hear, even though they share the relevant phonemes (Nielsen 2008:40f.).

Taken together, all these studies reveal how there is a close connection between speakers' production of words and their experience with those words in the input, even on a detailed level on an individual word basis.

As addressed in section 2, participants have greater difficulties recognizing words that have undergone a phonological change, and words that are in dense neighborhoods. A crucial part of these observations is that participants are also less accurate under these conditions, more often failing to recognize the words

\footnotetext{
${ }^{6}$ Enriching the models with fixed effects for vowel features (height, backness, rounding, length) did not alter any significant effects (or lacks thereof) reported in this section. Others measurements of ND were explored as well, such as a) the summed log frequency of the neighbors, b) neighbors sharing the same syntactic category as the target word. They differed little from the measurement used for ND above, and they did not improve the significance of ND in the analysis.
} 


\section{Neighborhood Density in Phonological Alternations}

altogether (Vitevitch \& Luce 1999:386f., Dirks et al. 2001:6ff., Vitevitch 2002:415). Relating these facts to Norwegian retroflexion, this means that speakers run a higher risk of not successfully recognizing a retroflex token of a word in a dense neighborhood than they do with a retroflex token in a sparse neighborhood (cf. Pierrehumbert 2002:117, Wedel 2006:264). As a result, the perceived distribution of retroflex tokens for $/ \mathrm{s} /$-words in dense neighborhoods will be slightly different compared with /s/-words in sparse neighborhoods, as there will be more solid evidence that retroflexion applies to the latter words than to the former words. In Goldinger (1998:257ff., 2000:156f.), participants unconsciously mimicked phonetic properties of words they heard several repetitions of significantly more than they did for words they heard fewer repetitions of. If speakers of Norwegian encounter retroflex tokens of $/ \mathrm{s} /$-words in sparse neighborhoods more often than in dense neighborhoods due to occasional failures in recognizing retroflex tokens in the latter category, then speakers could be reflecting this directly by producing more retroflex tokens in sparse neighborhoods than in dense neighborhoods, as illustrated in figure (12) above.

Moving on to the correlation between Onset and retroflexion, it is suggested in Stausland Johnsen (2009) that this link could be a result of the perceptual properties of the different/s/-onsets. Steriade $(2001: 222,2009: 164)$ claims that the likelihood of a phonological alternation is inversely related to the phonetic distance between the alternators, and Stausland Johnsen (2009) supports this view by finding that native Norwegians have greater difficulties distinguishing between $[\mathrm{stV}]$ and $[\mathrm{stV}]$ than between $[\mathrm{sV}]$ and $[\mathrm{sV}]$. In other words, the phonetic distance between $[\mathrm{sV}]$ and its retroflex counterpart $[\mathrm{sV}]$ is greater than between [stV] and its retroflex counterpart $[\mathrm{stV}]$. Since $[\mathrm{sV}]$ at the same time alternates less often with [sV] than [stV] does with [stV], as seen in figure (13), this supports the claim by Steriade that the phonetic distance between alternants stands in an inverse relation with their likelihood to alternate.

Experimental studies by Marslen-Wilson et al. (1996:1386f.) and Skoruppa et al. (to appear) show that when a derived form differs from its base in the initial consonant, the greater the phonetic distance between the forms is, the worse participants are at relating them to each other. Building on these observations, the link between phonetic distance in the onset and retroflexion rate can be explained in the same fashion as with ND above: Due to the greater phonetic distance between a base in $/ \mathrm{sV}-/$ and its retroflex variant [sV-], the retroflex [sV-] tokens run a greater risk of not being recognized as related to their base forms than would be the case for / $\mathrm{stV}$-/ words. Consequently, speakers would perceive more retroflex [stV-] tokens in relation to their base forms in $/ \mathrm{stV}^{-/}$than they would perceive retroflex [sV-] tokens in relation to base forms in $/ \mathrm{sV}-/$. Following the close connection between speakers' perceived input and own production as outlined above, they would replicate this skewed distribution in their own output.

With regard to the original connection between ND and retroflexion on the 
one hand, and between Onset and retroflexion on the other, as envisioned in this section, the occasional failures in recognizing retroflex tokens would probably only lead to a very small change in speakers' input. The significant difference observed in figure (12) and (13) would under this view represent the accumulated effect of this change, as the effect should be additive for each learner.

\section{$4 \quad$ Predicting Alternations for Novel Words}

When looking at the surface realization of words, it is not always clear which aspects of it are due to active and productive phonological operations in the mapping from representation to output, and which are due to lexicalized properties of the word which is realized. A standard procedure to tease these apart is to let speakers produce novel forms, as these by definition cannot have lexicalized properties.

A question that has not been addressed so far in the literature is what role ND is expected to play for the realization of novel words in alternations. Yet different accounts for the observed ND effects make different predictions for what the interaction between ND and novel words should be. According to Ussishkin \& Wedel (2009), the grammar suppresses alternations for words in dense neighborhoods in order to facilitate lexical access. This entails that when the grammar is ready to ship a word off to production, it estimates the ND of that word, and decides based on the outcome whether or not to apply a phonological operation to it. The same should therefore hold for novel words, as the grammar should be equally capable of making the same ND estimation for new words. According to the view advocated in section 3.2 above, however, the ND effect in alternations is the result of linguistic experience. Since speakers by definition have had no experience with novel words, this view predicts that no ND effect should be found for novel words. In order to test these opposing hypotheses, an experiment with nonce forms was designed.

\section{$5 \quad$ Experiment 2}

To evaluate the role of ND and Onset for retroflexion in novel words, an experiment along the same lines of experiment 1 was designed, only this time exclusively with nonce nouns. Nine nonce monosyllabic nouns were selected for production, three in $/ \mathrm{sV} /$, three in $/ \mathrm{stV} /$, and three in $/ \mathrm{skV} /$, with the vowels $/ \mathrm{tz} /, / \mathrm{ur} /$, and /a: $/{ }^{7}$ These nine nonce nouns formed nominal compounds with the first element sommer 'summer', ending in the retroflex trigger $/ \mathrm{r} /$. The procedure and participants were the same as in experiment 1 .

\footnotetext{
${ }^{7}$ The form /sku:/ was replaced with /sko:/, using the other available back round vowel in Norwegian, since /sku:/ is a real Norwegian noun.
} 


\section{Neighborhood Density in Phonological Alternations}

$0.5 \%$ of the items were excluded due to erroneous and disfluent production, and a subsequent $1.13 \%$ were excluded due to disagreement between the two taggers. In the end, the data set contained 3340 trials. Overall, 54.07\% of the tokens underwent retroflexion, and every item in the experiment exhibited variation. The item with least retroflexion was /st:/ with $35 \%$, and the item with most retroflexion was /sks:/ with $72 \%$.

\section{$5.1 \quad$ Results}

Figure (14) shows the relation between ND and retroflexion, and figure (15) the relation between Onset and retroflexion. For comparison, figure (14) contains the plots and regression lines from both experiment 1 and 2 . The filled circles and the solid line represent the data from experiment 2 .

(14)

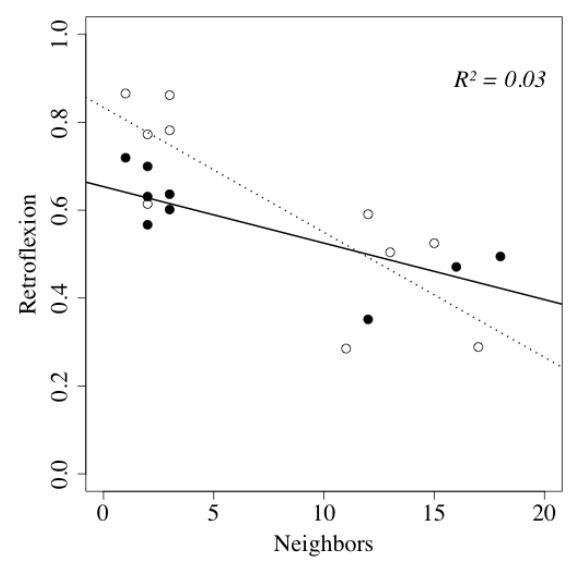

(15)

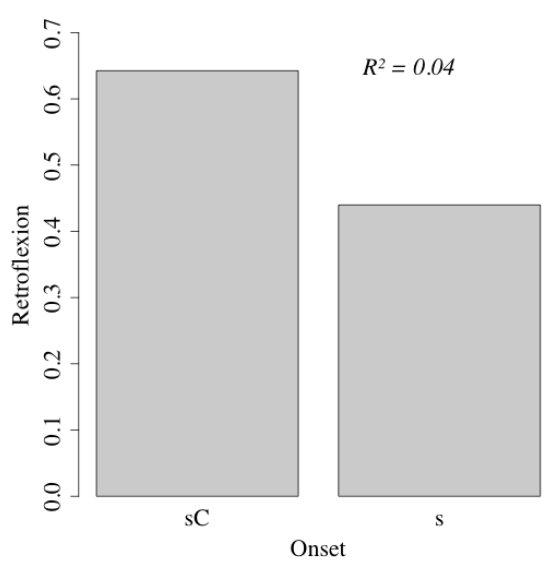

The results were analyzed with linear mixed effects models as for experiment 1 . Fixed effects were ND, Onset, Vowel, and various factors related to the experimental setting. When Onset was not included in the analysis, ND was a highly significant factor $\left(\chi^{2}(1)=14.49, p<.001\right)$, and similarly when ND was not included in the analysis, Onset was highly significant $\left(\chi^{2}(1)=20.57, p<.001\right){ }^{8}$ When the residuals of ND were added to a model with Onset included, no significant improvement was made $\left(\chi^{2}(1)=2.53, p=.11\right)$. When the residuals of Onset were added to a model with ND included, however, a significant improvement was seen $\left(\chi^{2}(1)=8.6, p<.01\right)$. This means that Onset explains the same data as ND does, but Onset also explains more, in the end leaving ND with no predictive power.

\footnotetext{
${ }^{8} / \mathrm{sk} /$ and $/ \mathrm{st} /$ did not behave differently in this experiment $\left(\chi^{2}(1)=2.63, p=.11\right)$, so they were collapsed as ' $\mathrm{sC}$ '.
} 
Sverre Stausland Johnsen

\subsection{Discussion}

The results from experiment 2 support the view of ND given in section 3.2, according to which ND might affect phonological alternations through the accumulated effect of having missed phonologically altered tokens in dense neighborhoods. Since novel words have not been experienced by speakers before, ND should have no impact on their alternations. The same prediction should follow from the exemplar models in Pierrehumbert (2002:114ff.) and Wedel (2006:254). In these models, producing an existing word involves sampling from a cloud of stored memories of perceived tokens of that word. Since ND affects how tokens are perceived, then ND will influence how tokens are produced. Given that novel words have not been perceived before, ND effects are predicted not to be found. This prediction is partly confirmed by Stephenson (2004), who in an explicit comparison of ND effects in existing and novel words found significantly less vowel dispersion for novel words, which she concludes is supportive of an exemplar model (2004:368). The lack of ND effects in experiment 2 serves as additional support for such models.

The results do not support the model put forward in Ussishkin \& Wedel (2009), where the grammar suppresses alternations for words in dense neighborhoods. Since the ND of a word bears no relation to the history of the word, their model should hold for existing and novel words alike. Whereas the results from experiment 1 admit the possibility of a link between ND and phonological alternations for existing words, the results from experiment 2 deny the same link for novel words. The lack of this link is also an expected result based on what has been observed elsewhere for the perception of novel words. If the speaker's grammar suppresses alternations in dense neighborhoods in order to facilitate lexical access, it would do so in order to facilitate lexical access for the listener. When a listener encounters a novel word, his task is to identify it as a novel word and learn it. Although a dense neighborhood inhibits quick and accurate recognition of existing words (see section 2 and 3.2), it has the opposite effect for novel words; it facilitates their learning (Storkel et al. 2006:1180). If functional pressure for efficient lexical access guides the inhibition of phonological alternations, then the grammar should in reality suppress such alternations in dense neighborhoods for existing words, but suppress them in sparse neighborhoods for novel words. Since there is no significant correlation in this direction in experiment 2 either, we are led to the conclusion that ND plays no active role in the mapping from representation to production.

This leaves the question of why Onset is a significant predictor of alternation for novel words. If there is a causal link between the perceptual properties of the onsets and their likelihood to alternate, as suggested in section 3.2, then this, too, is a word-specific property connected with words with a history, something novel word do not have. It has been known since Berko (1958:160ff.), however, that 


\section{Neighborhood Density in Phonological Alternations}

speakers are prone to generalize properties of individual words to phonological categories, which is seen precisely in speakers' treatment of novel words (see e.g. Finley \& Badecker 2009). That the category distinction between simple and complex word onsets is something speakers generalize over can be seen from processes in real languages. A well-known example comes from traditional Finnish, which inherited from its proto-language only words beginning with a vowel or a simple onset. Despite the abundance of word-internal consonant clusters in Finnish, the lack of initial clusters, which up until this point was simply a fact about existing words, was generalized to novel words as they were borrowed from other languages, as seen in borrowings such as koulu 'school' and ressi 'stress' (Suomi et al. 2008:55f.).

Note, however, that the same generalization from words to categories could have taken place if ND were the more dominant force behind the skewed distribution of retroflexion for existing words. Since there is such a strong correlation between ND and Onset, words in simple /s-/ and /sC-/ would under either scenario exhibit the same skewed distribution. The fact that this behavior is generalized to new words does not tell us what the dominant underlying cause for the pattern in real words is. Although the results from experiment 2 allow us to reject ND as a relevant factor for alternations in novel words, it does not extend to the results for experiment 1 . We can therefore not refute the possibility that ND plays a significant role for phonological alternations in existing words.

\section{Conclusion}

This paper has investigated the connection between neighborhood density (ND) and retroflex alternation in Norwegian. According to Ussishkin \& Wedel (2009), phonological alternations are suppressed in dense neighborhoods, but such a connection cannot be established for the retroflex alternation. In experiment 1 , where the retroflexion rate for real words was tested, the retroflexion is predicted just as well by the onset structure of the word as by the ND of the word. In experiment 2, on the other hand, where the retroflexion rate for novel words was tested, the onset structure of the word is a significant predictor of retroflexion, whereas ND is not.

Based on studies showing how ND affects the recognition of words, and studies showing how speakers' phonological production is directly influenced by what they have perceived, this paper offers an account for why ND can affect phonological alternations for existing words, but not for new words: If speakers more often fail to recognize alternated tokens in dense neighborhoods, then such alternated tokens will appear less frequently in speakers' own production, due to the direct connection between perceived input and produced output. Since new words have never been perceived before, ND cannot have caused an asymmetry in their recognition, and it should therefore not correlate with the produced output. 
Sverre Stausland Johnsen

In conclusion, ND might affect the phonological representation of an existing word, but it does not play a role in the mapping from representation to surface realization.

\section{References}

Baayen, R. H., D. J. Davidson, and D. M. Bates. 2008. Mixed-Effects Modeling with Crossed Random Effects for Subjects and Items. Journal of Memory and Language 59:390-412.

Bates, Douglas, [bates at stat.wisc.edu] "Testing Fixed Effects in Binomial lmer...Again?" In R Special Interest Group on Mixed Effect Models, Notably lmer() Related. [r-sig-mixed-models at r-project.org] 8 January 2008; Internet.

Bates, Douglas and Martin Maechler. 2010. lme4: Linear Mixed-Effects Models Using S4 Classes. R Package Version 0.999375-33. http:/CRAN.Rproject.org/package $=1 \mathrm{me} 4$

Becker, Michael and Andrew Nevins. 2009. Initial-Syllable Faithfulness as the Best Model of Word-Size Effects in Alternations. Paper presented at NELS 40, November 13-15, in Cambridge, MA.

Berko, Jean. 1958. The Child's Learning of English Morphology. Word 14:150177.

Dirks, Donald D., Sumiko Takayanagi, Anahita Moshfegh, P. Douglas Noffsinger, and Stephen A. Fausti. 2001. Examination of the Neighborhood Activation Theory in Normal and Hearing-Impaired Listeners. Ear \& Hearing 22:1-13.

Eliasson, Stig. 1986. Sandhi in Peninsular Scandinavian. In H. Andersen, ed., Sandhi Phenomena in the Languages of Europe, 271-300, Berlin: Mouton de Gruyter.

Finley, Sara and William Badecker. 2009. Artificial Language Learning and Feature-Based Generalization. Journal of Memory and Language 61:423-437.

Goldinger, Stephen. 1998. Echoes of Echoes? An Episodic Theory of Lexical Access. Psychological Review 105:251-279. 


\section{Neighborhood Density in Phonological Alternations}

Goldinger, Stephen D. 2000. The Role of Perceptual Episodes in Lexical Processing. In A. Cutler, J. McQueen, and R. Zondervan, eds., Proceedings of SWAP - Spoken Word Access Processes, 155-158, Nijmegen: Max-Planck Institute for Psycholinguistics.

Harrington, Jonathan, Sallyanne Palethorpe, and Catherine I. Watson. 2000. Does the Queen Speak the Queen's English? Nature 408:927-928.

Jaeger, T. Florian. 2008. Categorical Data Analysis: Away from ANOVAs (Transformation or Not) and Towards Logit Mixed Models. Journal of Memory and Language 59:434-446.

Järvikivi, Juhani, Pirita Pyykkönen, and Jussi Niemi. 2009. Exploiting Degrees of Inflectional Ambiguity: Stem Form and the Time Course of Morphological Processing. Journal of Experimental Psychology: Learning, Memory, and Cognition 35:221-237.

Kristoffersen, Gjert. 2000. The Phonology of Norwegian. Oxford: Oxford University Press

Luce, Paul A. and David B. Pisoni. 1998. Recognizing Spoken Words: The Neighborhood Activation Model. Ear \& Hearing 19:1-36.

Marslen-Wilson, William, Helen E. Moss, and Stef van Helen. 1996. Perceptual Distance and Competition in Lexical Access. Journal of Experimental Psychology: Human Perception and Performance 22:1376-1392.

Moen, Inger, Hanne Gram Simonsen, Arne Martinus Lindstad, and Steve Cowen. 2003. The Articulation of the East Norwegian Apical Liquids / $/ \mathrm{r}$. Proceedings of the $15^{\text {th }}$ International Congress of Phonetic Sciences, Barcelona 2003, 1755-1758, Adelaide: Causal Productions.

Munson, Benjamin and Nancy Pearl Solomon. 2004. The Effect of Phonological Neighborhood Density on Vowel Articulation. Journal of Speech, Language, and Hearing Research 47:1048-1058.

Nielsen, Kuniko Yasu. 2008. Word-Level and Feature-Level Effects in Phonetic Imitation. Ph.D. thesis, University of California, Los Angeles, CA.

Pierrehumbert, Janet B. 2002. Word-Specific Phonetics. In C. Gussenhoven and N. Warner, eds., Laboratory Phonology 7, 101-139, Berlin: Mouton de Gruyter. 
Sverre Stausland Johnsen

Pinheiro, José C. and Douglas M. Bates. 2000. Mixed-Effects Models in S and SPLUS. New York: Springer Verlag.

Pycha, Anne, Sharon Inkelas, and Ronald Sprouse. 2007. Morphophonemics and the Lexicon. A Case Study from Turkish. In M.-J. Solé, P. S. Beddor, and M. Ohala, eds., Experimental Approaches to Phonology, 369-385, Oxford: Oxford University Press.

R Development Core Team. 2010. R: A Language and Environment for Statistical Computing. R Foundation for Statistical Computing, Vienna, Austria. ISBN 3900051-07-0, URL http://www.R-project.org.

Sankoff, Gillian and Hélène Blondeau. 2007. Language Change across the Lifespan: /r/ in Montreal French. Language 83:560-588.

Simonsen, Hanne Gram and Inger Moen. 2004. On the Distinction between Norwegian / $/$ and /ç/ from a Phonetic Perspective. Clinical Linguistics \& Phonetics 18:605-620.

Skoruppa, Katrin, Anna Lambrechts, and Sharon Peperkamp. (To appear). The Role of Phonetic Distance in the Acquisition of Phonological Alternations. Proceedings of NELS 39.

Stausland Johnsen, Sverre. 2009. Perceptual Distance in Norwegian Retroflexion. Paper presented at NELS 40, November 13-15, in Cambridge, MA.

Stephenson, Lisa. 2004. Lexical Frequency and Neighborhood Density Effects on Vowel Production in Words and Nonwords. Proceedings of the $10^{\text {th }}$ Australian International Conference on Speech Science \& Technology, 364-369, Australian Speech Science \& Technology Association Inc.

Steriade, Donca. 2001. Directional Asymmetries in Place Assimilation. A Perceptual Account. In E. Hume and K. Johnson, eds., The Role of Speech Perception in Phonology, 219-250, San Diego, CA: Academic Press.

Steriade, Donca. 2009. The Phonology of Perceptibility Effects: The P-Map and its Consequence for Constraint Interaction. In K. Hanson and S. Inkelas, eds., The Nature of the Word. Studies in Honor of Paul Kiparsky, 151-179, Cambridge, MA: The MIT Press. 


\section{Neighborhood Density in Phonological Alternations}

Storkel, Holly L., Jonna Armbrüster, and Tiffany P. Hogan. 2006. Differentiating Phonetic Probability and Neighborhood Density in Adult Word Learning. Journal of Speech, Language, and Hearing Research 49:1175-1192.

Suomi, Kari, Juhani Toivanen, and Riikka Ylitalo. 2008. Finnish Sound Structure. Phonetics, Phonology, Phonotactics and Prosody. Oulu: Oulu University Press.

Torp, Arne. 2007. $R$ - ei urokråke i språket. Oslo: Det norske samlaget.

Tsapkini, Kyrana, Eva Kehayia, and Gonia Jarema. 1999. Does Phonological Change Play a Role in the Recognition of Derived Forms across Modalities? Brain and Language 68:318-323.

Ussishkin, Adam and Andrew Wedel. 2009. Lexical Access, Effective Contrast, and Patterns in the Lexicon. In P. Boersma and S. Hamann, eds., Phonology in Perception, 267-292, Berlin: Mouton de Gruyter.

Vitevitch, Michael S. 2002. Naturalistic and Experimental Analyses of Word Frequency and Neighborhood Density Effects in Slips of the Ear. Language and Speech 2002:407-434.

Vitevitch, Michael S. and Paul A. Luce. 1999. Probabilistic Phonotactics and Neighborhood Activation in Spoken Word Recognition. Journal of Memory and Language 40:374-408.

Wedel, Andrew B. 2006. Exemplar Models, Evolution, and Language Change. The Linguistic Review 23:247-274.

Wright, Richard. 2003. Factors of Lexical Competition in Vowel Articulation. In J. Local, R. Ogden, and R. Temple, eds., Phonetic Interpretation. Papers in Laboratory Phonology VI, 75-86, Cambridge: Cambridge University Press.

Sverre Stausland Johnsen

Department of Linguistics

Boylston Hall $3^{\text {rd }}$ floor

Harvard University

Cambridge, MA 02138

johnsen@fas.harvard.edu 